\title{
BROWSER'S NOTES
}

\section{Browser's notes}

Published online: 22 January 2018

(C) ISS 2018

\section{Effect of CT on management plan in malleolar ankle fractures.}

Kumar A, et al.

Foot Ankle Int. (2018); 39(1):59-66

\section{Impact of computed tomography on operative planning} for ankle fractures involving the posterior malleolus.

Donohoe $S$, et al.

Foot Ankle Int. (2017); 38(12):1337-42

Two independent articles assessed the role of CT in operative decisions for patients with malleolar ankle fractures. Kumar, et al. prospectively evaluated 56 patients (mean age 34.7 years, $86 \%$ male) with radiography and CT. AO/Weber fracture type was first identified using radiographs and a treatment plan was determined by 3 orthopedic surgeons. Thereafter, the CT was reviewed to determine changes in fracture evaluation and treatment plan. CT evaluation changed the operative management plan in $23 \%(13 / 56)$ of the cases. The treatment plan changed for $6(40 \%)$ of the 15 type C and 6 (29\%) of 21 type B fractures, but only 1 of the 20 type A fractures. An additional anterolateral tibial (Chaput) fragment was identified only by CT in 4 patients (7\%). Of 13 posterior malleolar fractures, $6(46 \%)$ had a change in treatment plan. The authors indicate CT evaluation should be considered for all type B and type C ankle fractures. In a separate study, Donohoe, et al., retrospectively reviewed 3,394 ankle fractures and finding 376 (11\%) with posterior malleolar involvement identified by radiographs; 25 $(6.6 \%)$ of these patients (mean age 37.8 years, $56 \%$ female) had both radiographs and CT available and became the study group. Fractures were evaluated separately on radiographs and CT by 3 orthopedic surgeons in blinded fashion for frac- ture type (simple or complex/comminuted), treatment approach, and, if indicated, operative plan. With CT considered the standard, 14/25 (56\%) of ankle fractures were complex, however, 8 of these were incorrectly categorized as simple by radiographs; 5 of 11 simple fractures were incorrectly called complex by radiographs. In $16 \%$ of cases the decision to operate was changed by CT evaluation, and patient positioning and operative approach were altered in 44\%. CT should play an important role in treatment planning for patients with posterior malleolar fractures.

Proximal radial head fractures in young gymnasts: A case series of newly described overuse injuries.

Santelli J, et al.

Clin J Sport Med. (2017); Oct 11. [Epub ahead of print] PMID: 2902375

While stress-related injuries of the distal radius in gymnasts are well described, injuries of the proximal radius have been considered rare. Three competitive gymnasts, ages 11 and 12, presented with several weeks to months of elbow pain one bilateral, that was reproducible on physical exam. None of the patients reported an acute injury. All had a Salter IV fracture of the proximal radius involving the radial head epiphysis. Radiographs demonstrated irregularities, fissuring, or fracture of the radial head. MR imaging better demonstrated the fractures with associated marrow edema-like signal. These fractures can be differentiated from epiphyseal clefts by the abnormal marrow signal on MR images.

\section{Abstracted by C. S. Winalski, M.D.}

April 2018 